\title{
PICK-UP RATE OF SUSPENDED SAND DUE TO TSUNAMI
}

\author{
Takumi Yoshii $^{1}$, Masaaki Ikeno ${ }^{1}$, Masafumi Matsuyama² and Naoki Fujii ${ }^{3}$
}

\begin{abstract}
The pick-up rate formula which can apply to wide-range of grain size was presented through dimensional analysis and laboratory experiments. The result of the dimensional analysis revealed that there are three must-considered nondimensional parameters: "non-dimensional sand grain diameter", "non-dimensional settling velocity", and "nondimensional shear stress". The pick-up rate formula which considers these parameters can predict the pick-up rate of $0.08 \mathrm{~mm}$ and $0.2 \mathrm{~mm}$ grains measured in the experiment with sufficient accuracy. The topography change around a harbor due to tsunami was measured in the laboratory experiment and compared with the result of numerical simulations. The numerical simulation with the presented pick-up rate formula shows a better agreement with the laboratory experiment than that with Takahashi et al. (2000)'s model.
\end{abstract}

Keywords: tsunami; sediment transport; pick-up rate

\section{Introduction}

Tsunami has caused devastating damage to the coastal region in recent decades. Coastal structures were destroyed due to the enormous wave force of Tsunami. In order to minimize damage and protect life environment it is important to protect the absolutely essential infrastructures such as electric power plants from a tsunami.

Tsunami can carry the large amount of sea sand on the harbor and the deposition and/or erosion on the harbor can reduce the port function (Takahashi et al, 2000; Nishihata et al, 2006). The deposition of the sediment in the harbors where power plants were located may affect the function of a cooling system by preventing water intake. Therefore, the prediction of the sediment transport due to tsunami plays an essential role in safety assessment and revamping program of the harbor structures against tsunami.

Several sediment transport models have been developed in order to predict seabed topography change due to tsunami (e.g., Takahashi et al., 2000). Most of them were derived using the laboratory experiment with steady flow in order to represent the high shear stress on the sea bed. However, the authors have revealed the importance of the unsteady process in the sediment transport due to tsunami through the laboratory experiment with a wave tank and solitary waves (Yoshii et al., 2009).

To include the unsteady process of sediment transport into numerical model, Takahashi et al. (2000) introduced the pick-up rate and settling rate between the bed load layer and the suspended load layer into sediment transport model (Figure 1). In the two layered sediment transported model, the suspended load is supplied through the pick-up rate from the bottom and reduced through the settling rate. Therefore, the amount of the suspended load depends not on the local flow but on the history of the flow. Takahashi et al. (2000) reported that the two layered sediment transport model was successful in representing the unsteady process in the sediment transport. The two layered model can reproduce the time-lags between the velocity and the sediment concentration.

In order to apply the two layered model to a tsunami, it is necessary to calculate the pick-up rate due to the tsunami. Takahashi et al. (2000) have derived the pick-up rate formula which can be applied to a tsunami through the laboratory experiment with $0.2 \mathrm{~mm}$ grain size. The proposed formula is expressed by

$$
P / \sqrt{s g d}=0.012 \psi^{2}
$$

where $s$ is the density of the sand in water, $g$ is gravity acceleration, $d$ is grain size and $\psi$ is Shield's number, the non-dimensional parameter which represents the shear stress on the bottom, respectively. This formula depends only on Shield's number. However, in view of the fact that the upward current must be larger than the settling velocity of the sand in the pick-up process, the neglect of the effect of settling velocity in the pick-up rate formula is believed to reduce its applicability to wide-range grain

\footnotetext{
${ }^{1}$ Environmental Science Research Laboratory, Central Research Institute of Electric Power Industry, 1646 Abiko, Abiko, Chiba, 270-1194, Japan

2 Civil Engineering Research Laboratory, Central Research Institute of Electric Power Industry, 1646 Abiko, Abiko, Chiba, 270-1194, Japan

3 Civil Engineering Operation Center, Tokyo Electric Power Services Co., Ltd, 3-3-3 Higashi-Ueno, Taito, Tokyo, 110-0015, Japan
} 
size because grain size significantly varies the settling velocity of the sand. Therefore, there is a need for improvement to apply to wide range of grain sizes.

In this study, the authors propose a new pickup rate formula which takes account of the effect of the sand grain size through dimensional analysis and the experimental results by Yoshii et al. (2009). The topography change around the harbor due to tsunami was measured in an experiment. To validate the proposed formula, the comparison between the measured topography and the calculated topography using the proposed formula was conducted.

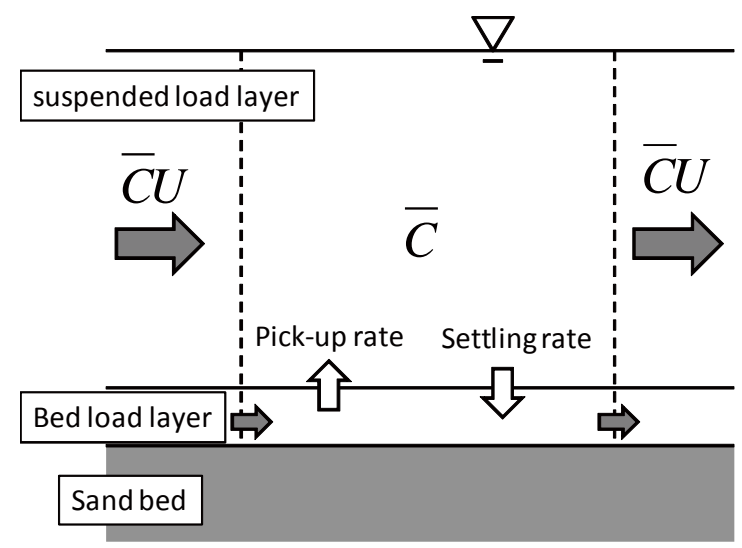

Figure 1. Sketch of the two layered sediment transport model. The sediment transport was divided into the suspended load layer and bed load layer. Bed load directly respond to the shear stress on the bed. The suspended load was supplied and reduced through the pick-up rate from the bottom and settling of the sand.

\section{Dimensional analysis}

To obtain the pick-up rate formula which can be applied to various sand grain sizes, the authors investigated the dominant parameters that can account for the difference in pick-up rates of various sediment sizes using a dimensional analysis. First, we chose the following dominant parameters in the sediment transport: friction velocity at the bottom $\left(u_{b}^{*}\right)$, density of sediment $\left(\rho_{s}\right)$, density of the fluid $\left(\rho_{w}\right)$, kinematic viscosity coefficient $(v)$, gravity acceleration $(g)$, sand grain size $(d)$, settling velocity of the sand $\left(w_{s}\right)$. Using these parameters, the pick-up rate can be expressed by

$$
P=k u_{b}{ }^{n 1} \rho_{s}^{n 2} \rho_{w}^{n 3} g^{n 4} d^{n 5} v^{n 6} w_{s}^{n 7}
$$

where $k$ is non-dimensional coefficient and $n_{j}$ is constant number of each component. Since the dimension of length, mass, time must be equal to each side, the constant numbers is given by

$$
\begin{gathered}
n_{1}-3 n_{2}-3 n_{3}+n_{4}+n_{5}+2 n_{6}+n_{7}=1 \quad \text { (for length) } \\
n_{2}+n_{3}=0 \quad \text { (for mass) } \\
-n_{1}-2 n_{4}-n_{6}-n_{7}=0 \quad \text { (for time) }
\end{gathered}
$$

Solving these equations reduces seven unknown constant numbers to four. The substitution of $n_{3}, n_{4}, n_{5}$ by $n_{1}, n_{2}, n_{6}, n_{7}$ leads the following equation:

$$
P=k g^{1 / 2} d^{1 / 2} s^{n_{2}}\left\{u_{b} * /(g d)^{1 / 2}\right\}^{n_{1}}\left\{v^{2} /\left(g d^{3}\right)\right\}^{n_{6}}\left\{w_{s} /(g d)^{1 / 2}\right\}^{n_{7}}
$$

where, $s$ means the density of the sand in water $\left(=\rho_{\mathrm{s}} / \rho_{w}\right)$. Dividing both side by $\mathrm{g}^{1 / 2} \mathrm{~d}^{1 / 2} \mathrm{~g}^{1 / 2}$ derives

$$
P / \sqrt{s g d}=F(s)\left\{u_{b} *^{2} /(s g d)\right\}^{A_{1}}\left\{v^{2} /\left(s g d^{3}\right)\right\}^{A_{2}}\left(w_{s} / \sqrt{s g d}\right)^{A_{3}},
$$

where $A_{1}, A_{2}, A_{3}$ are the coefficients which include $n_{l}, n_{6}, n_{7}, n_{2}$, and $F(\mathrm{~s})$ means the function of $s$. It is well known that there is a critical friction velocity at which the movement of bed material starts. Based on the idea, there must be the critical friction velocity $\left(u_{c r}{ }^{*}\right)$ which the pick-up process starts and the velocity component over the critical friction velocity $\left(u_{e}^{*}\right)$ should affect the pick-up of sands. Since sand movement depends on the square of the friction velocity, the actual friction velocity on the bottom is derived by 


$$
u_{e} *^{2}=u_{b} *^{2}-u_{c r} *^{2} .
$$

Using $u_{\mathrm{e}} *$ instead of bottom friction $\left(u_{\mathrm{b}} *\right)$, the following equation is derived.

$$
P / \sqrt{s g d}=F(s)\left\{\left(u_{b} *^{2}-u_{c r} *^{2}\right) /(s g d)\right\}^{A_{1}}\left\{v^{2} /\left(s g d^{3}\right)\right\}^{A_{2}}\left(w_{s} / \sqrt{s g d}\right)^{A_{3}} .
$$

By substituting Shield's number ( $\psi$ ) for $u * / s g d$, we finally get the pick-up rate formula expressed by,

$$
P / \sqrt{s g d}=F(s)\left(\psi-\psi_{c r}\right)^{A_{1}}\left\{v^{2} /(s g d)^{3}\right\}^{A_{2}}\left(w_{s} / \sqrt{s g d}\right)^{A_{3}} .
$$

The obtained pick-up rate formula is composed of three non-dimensional parameters: 'nondimensional sand grain diameter' $\left(s g d^{3} / v^{2}\right)$ and 'non-dimensional settling velocity' $\left(w_{s} /(s g d)^{1 / 2}\right)$ in addition to Shield's number of 'non-dimensional shear stress' $\left(u^{* 2} / s g d\right)$. It means that these three parameters can account for the effect of sand grain size in the pick-up process. The function of s $(F(s))$ is believed to be almost constant because the variation of the density of actual sand is not large. As a result, these three non-dimensional components play a very important roll to present the difference in the pick-up rate between various grains.

\section{Experiment in the pick-up rate due to tsunami}

To check the availability of the proposed pick-up rate formula, the pick-up rates of $0.08 \mathrm{~mm}$ and 0.2 $\mathrm{mm}$ grains due to the tsunami passing the horizontal sand bed without wave-splitting or wave-breaking were estimated using the experimental result of Yoshii et al.(2009). The experiment had been conducted using the two dimensional flume with piston-type wave generator. The vertical distribution of the horizontal velocity and the sediment concentration had been measured at the point where the sediment transport was considered not to be affected by the length of the sand bed (Figure 2). The maximum Shield's number in the experiment was around 1.

To obtain the pick-up rate from the measured data, the authors used the one-dimensional sediment transport equation expressed by

$$
\frac{\partial}{\partial t} \int_{z_{b}}^{\eta+h} c(z) d z+\frac{\partial}{\partial x} \int_{z_{b}}^{\eta+h} c(z) u(z) d z=P-w_{s} c_{b}
$$

where, $c, c_{b}, u, w_{s}, P$ express the concentration of the suspended sediment, the suspended sediment concentration at the bottom of the suspended load layer, the horizontal velocity, the settling velocity of the sediment and the pick-up rate from the sand bed, respectively. The direction of $x$ is the same way of the tsunami propagates, $z$ means the vertical distance from the bottom and $t$ means time, respectively.

When a wave progresses over a flat bed without deformation of its shape, the time history of the
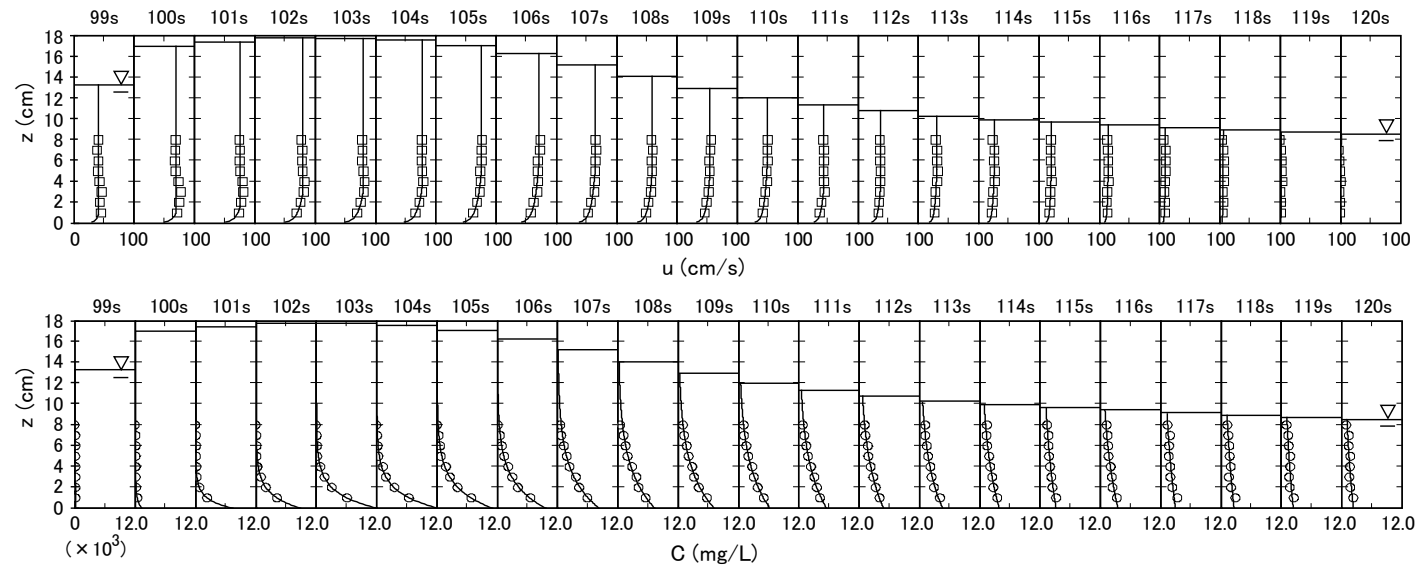

Figure 2. Vertical profiles of the measured horizontal velocity (top) and suspended sediment concentration (bottom). The horizontal line in the figures indicates the water level. The water elevation o the incident wave is $6 \mathrm{~cm}$ and the grain size is $0.08 \mathrm{~mm}$. The marks plotted in these figures indicate the measured data and the solid lines present the approximated vertical profiles (after Yoshii et al, 2009). 
momentum flux is constant at all the places even though there is time-lag in the time history between different places. Furthermore, if the length of the sand floor doesn't affect the sediment flux, the time history of the sediment flux is also assumed to be constant. Since the time-lag between different places is related to the wave celerity, the spatial-difference of the flux can be represented as the timedifference with wave celerity, which is expressed by

$$
\frac{\partial F}{\partial x}=-\frac{1}{w_{c}} \frac{\partial F}{\partial t},
$$

where, $F$ and $w_{c}$ mean the flux of the sediment and wave celerity, respectively. The limited data measured at $1 \mathrm{~m}$ away from the measuring point of vertical distribution shows almost the same timehistory with that measured. Therefore, the assumption presented as equation 12 is believed to be effective in this experience. By the introduction of equation 12 into equation 11, finally, we get the following equation,

$$
P=\frac{\partial}{\partial t} \int_{z_{b}}^{\eta+h} c(z) d z-\frac{\partial}{w_{c} \partial t} \int_{z_{b}}^{\eta+h} c(z) u(z) d z+w_{s} c_{b}
$$

The pick-up rate was calculated from the experimental result using equation 13.

According to Yoshii et al. (2009), the vertical distribution of the horizontal velocity can be approximated with logarithmic function and the vertical distribution of the suspended sediment concentration can be expressed with power function (Figure 2). Therefore, the authors calculated the integral terms of the horizontal velocity and the suspended sediment concentration using the approximated vertical distribution acquired by these functions respectively. The settling velocity was calculated using equation 14 (Rubey, 1933);

$$
w_{s}=\sqrt{\frac{2}{3} s g d+\frac{36 v^{2}}{d^{2}}}-\frac{6 v}{d}
$$

To calculate the wave celerity, the wave celerity was checked using the water levels measured at the same point where the concentration and velocity were measured and at the point where is $6.0 \mathrm{~m}$ away from that point. From the result, we discovered that the wave celerity was calculated with sufficient accuracy using the following equation;

$$
w_{c}=\sqrt{g h}\left[1+1.3\left(\frac{\eta}{h}\right)^{0.85}\right]
$$

where $h$ is the static water depth and $\eta$ is the water elevation.

Figure 3 shows the pick-up rate of $0.08 \mathrm{~mm}$ and $0.20 \mathrm{~mm}$ grains obtained from the experiment with Shield's number. To reduce the noise component, the moving average of $0.2 \mathrm{~s}$ was applied to the time history of the measured data. The incident waves presented in figure 3 were equivalent to each other. The pick-up rate varies between at the acceleration time and at deceleration time (Figure 3 ). The pickup rate at the acceleration is much lower than that at the deceleration time. This characteristic feature appears in the both cases. Furthermore, the pick-up rates of $0.08 \mathrm{~mm}$ sand and $0.2 \mathrm{~mm}$ sand contradicted each other in spite of the same Shield's number. The predicted pick-up rate by Takahashi et al. (2000) presented as the broken lines are relatively consistent with the measured pick-up rate of 0.2 $\mathrm{mm}$ sand at the deceleration time. However, the predicted pick-up rate disagrees with that of $0.08 \mathrm{~mm}$ sand.

The difference in pick-up rate between at the acceleration time and at the deceleration time indicates that the pick-up rate can vary with acceleration of the flow. The predicted pick-up rate using Takahashi et al. (2000)'s formula, which is based on the experiment with steady flow relatively agrees with the pick-up rate measured at the deceleration time. This result shows that the process of pick-up in the acceleration time might differ from that reported in the past literatures. However, the acceleration time in the experiment is rather limited and the clearance between the bed floor and the measurement instrument set at the lowest position in the measurement is insufficient to observe the initial process of sediment pick-up. Therefore, it was insufficient data for further investigation of the effect of 
acceleration to the pick-up rate. For that reason, this study deals focuses on the pick-up rate measured at the deceleration time.

The disagreement between the measured pick-up rate and the predicted pick-up rate implies that there is the effect due to the sand grain size changes on the pick-up rate and that is not included sufficiently in the conventional pick-up rate formulae which depend only on Shield's number. Shield's number basically represents the effect of shear stress on the bottom though it includes the sand grain size. Therefore, this result means that the pick-up of sediment does not depend only on the shear stress at the bottom.

To apply the presented pick-up rate formula (equation 10) to the measured pick-up rate, equation 10 was transformed into the following equation and the coefficients of $A_{2}, A_{3} / A_{1}$ were determined by fitting the predicted results to the experimental results.

$$
P / \sqrt{s g d}\left\{v^{2} /(s g d)^{3}\right\}^{-A_{2}}=F(s)\left\{\left(\psi-\psi_{c r}\right)\left(w_{s} / \sqrt{s g d}\right)^{A_{3} / A_{1}}\right\}^{A_{1}} .
$$

The critical Shield's number which the pick-up of bottom sands starts was assumed to be the same with

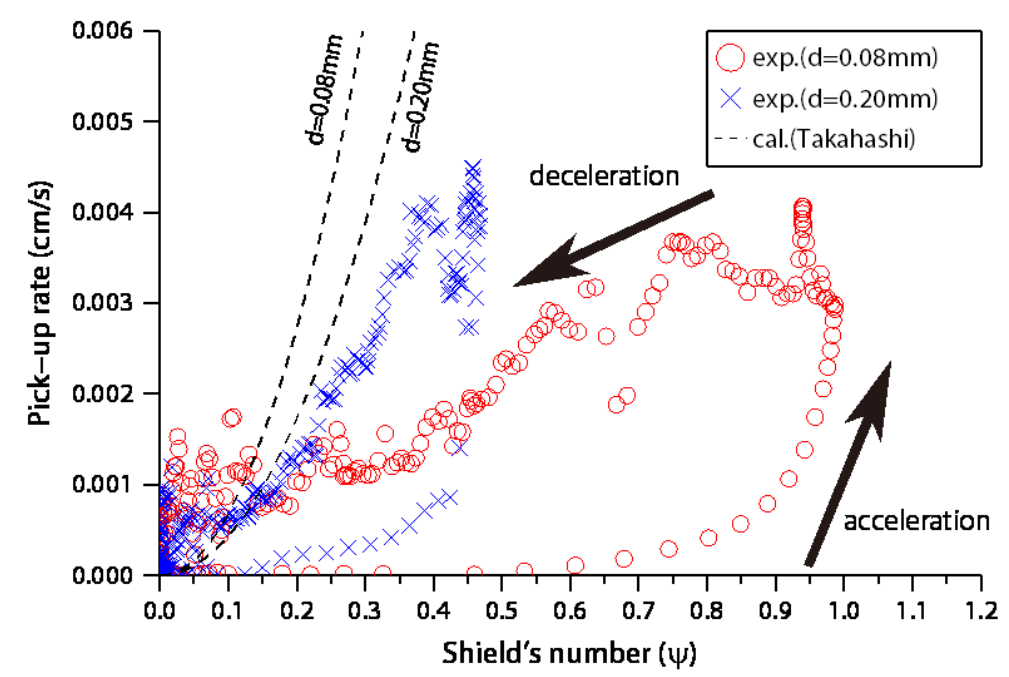

Figure 3. Pick-up rate of the $0.08 \mathrm{~mm}$ sand and $0.02 \mathrm{~mm}$ sand. The incident wave was same for both cases $(6.0 \mathrm{~cm})$. The dotted-line indicate the predicted pick-up rate calculated using Takahashi et al (2000)'s formula.

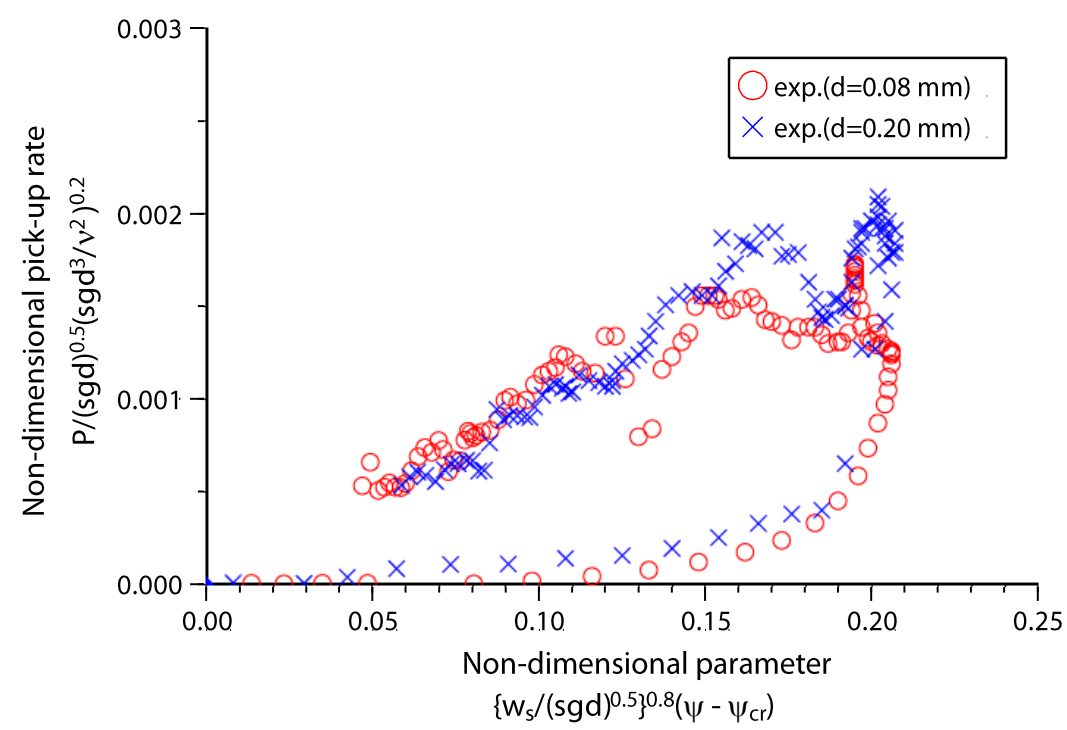

Figure 4. Pick-up rate of the $0.08 \mathrm{~mm}$ sand and $0.02 \mathrm{~mm}$ sand presented using the proposed nodimensional parameters. 


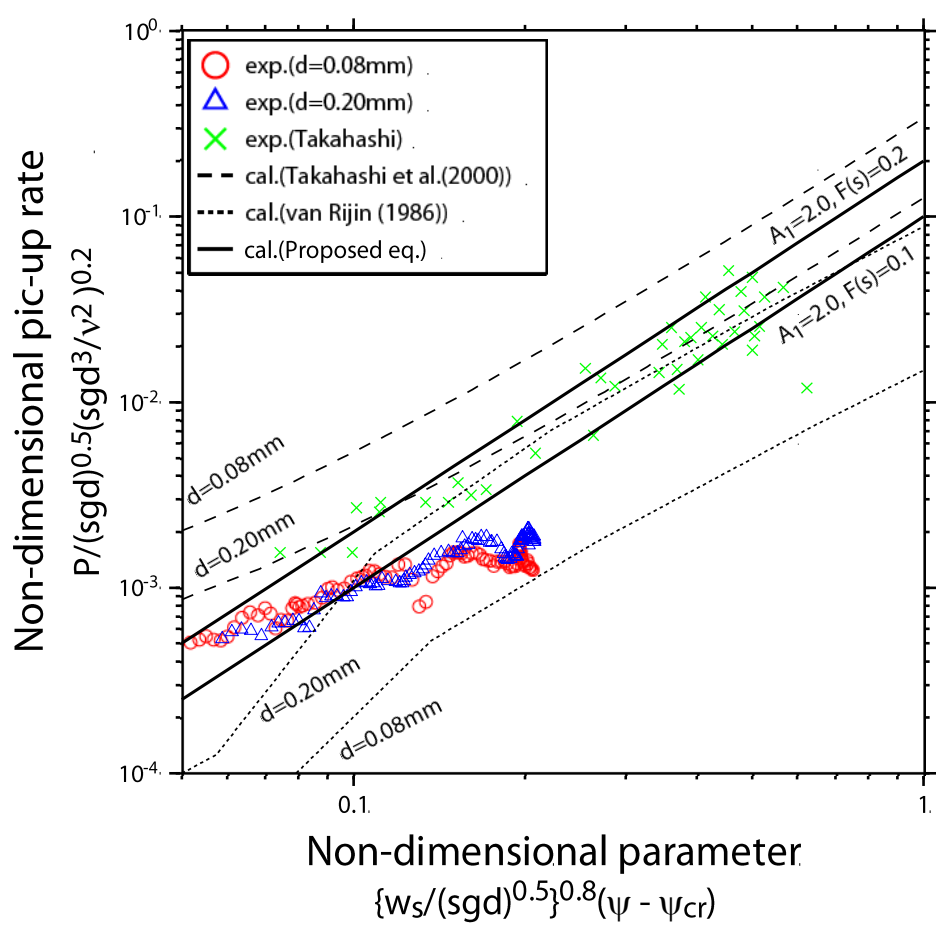

Figure 5. Comparison between the pick-up rate measured in this study and Takahashi et al. (2000) and the predicted pick-up rate. The dotted lines present the predicted pick-up rate using van Rijin (1986)'s formula. The broken lines indicate that using Takahashi et al. (2000)'s formula. The solid bold line indicates the predicted pick-up rate using the presented formula (equation 16).

the critical Shield's number which causes the bed load. As a result, the minimum Shield's number ( $\left.\psi_{\mathrm{cr}}\right)$ was calculated according to Iwagaki (1956). By fitting the equation to the measured data, the following coefficients were obtained: $A_{3} / A_{1}=0.8, A_{2}=-0.2$.

Figure 4 shows the measured pick-up rates using the proposed non-dimensional parameters. The pick-up rates of the both sands considerably consist with each other by introducing these nondimensional parameters. The inclusion of effect of settling velocity in the equation plays an important role in the correspondence of the pick-up rate of both sands. Since the kinematic viscosities are the same in both experiments and the effect of the grain size on the non-dimensional pick-up rate is little, the nondimensionalization of the pick-up rate does not show significant effect on the result.

Since the maximum Shield's number obtained in Yoshii et al. (2009) is less than that of tsunami, it is necessary to fit the pick-up data measured under high Shield's number as same level as actual tsunami to have applicability to a tsunami. Thus, the coefficients of $A_{1}$ were determined by fitting proposed equation to the experimental results by Takahashi et al. (2000) in addition to the result of Yoshii et al. (2009). Although the experiment had been conducted using the water tunnel and steady flow, Takahashi et al. (2000) had measured the pick-up rate under the condition that Shield's number ranged from 0.2 to 1.4. Figure 5 shows the pick-up rate measured by Yoshii et al. (2009) and Takahashi et al. (2000) with the presented non-dimensional parameters.

Using the proposed non-dimensional parameters, the pick-up rate of the different grain size under the wide-ranged shear stress shows an obvious upward trend (figure 5). The coefficient of $A_{1}$ seems to be 2.0 if linear relationship is adopted. The proposed formula can fit to the measured result with the coefficient of $F(\mathrm{~s})$ ranging from 0.1 to 0.2 . In the figure, the conventional pick-up rate formulae were also plotted as dotted lines and broken lines. The predicted pick-up rates using these formulae shows a satisfactorily good agreement with the measured pick-up rate of $0.2 \mathrm{~mm}$ sand. However, these results considerably disagree with that of $0.08 \mathrm{~mm}$ sand. Therefore, introducing these non-dimensional parameters in the pick-up rate formula can be applied to wide range of grain size compared to the conventional formulas. 
The experiments in the topography change around the harbor

To investigate the flow field and the consequent topography change due to tsunami around a harbor, a laboratory experiment was conducted and the necessary data for verification of numerical calculation was obtained.

The experiment was conducted using the wave flume of $58 \mathrm{~m}$ by $20 \mathrm{~m}$ (figure 6). The pump-type wave generator equipped with the flume enabled us to generate a long period wave compared to the piston-type one. The sand bed of $8 \mathrm{~m}$ by $8 \mathrm{~m}$ was set at the end of the flume. The model harbor with typical form was constructed on the movable bed of $0.08 \mathrm{~mm}$ sand and its size was approximately $6.0 \mathrm{~m}$ by $6.0 \mathrm{~m}$ (Figure 7). The breakwater of the harbor was $0.15 \mathrm{~m}$ thick. We generate the half part of a wave of 60 seconds as an incident wave. The maximum water elevation of the incident wave was $6 \mathrm{~cm}$ and the water depth at the generator was $78 \mathrm{~cm}$. The incident wave reaches the plane part where the

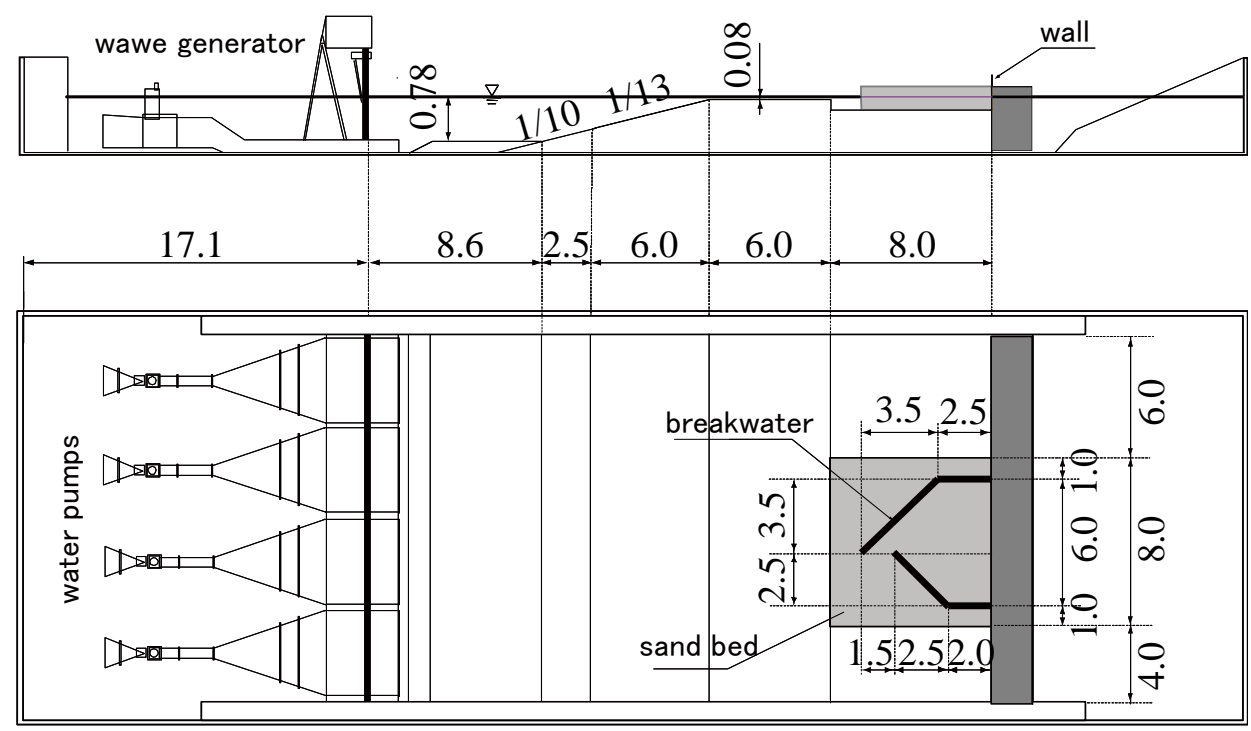

< unit: $\mathrm{m}$ >

Figure 6. Experimental setup. The grain size used in the sand bed was $0.08 \mathrm{~mm}$. The incident wave of $6 \mathrm{~cm}$ elevation and $30 \mathrm{~s}$ period which simulate the uprush of tsunami was generated by the wave generator.

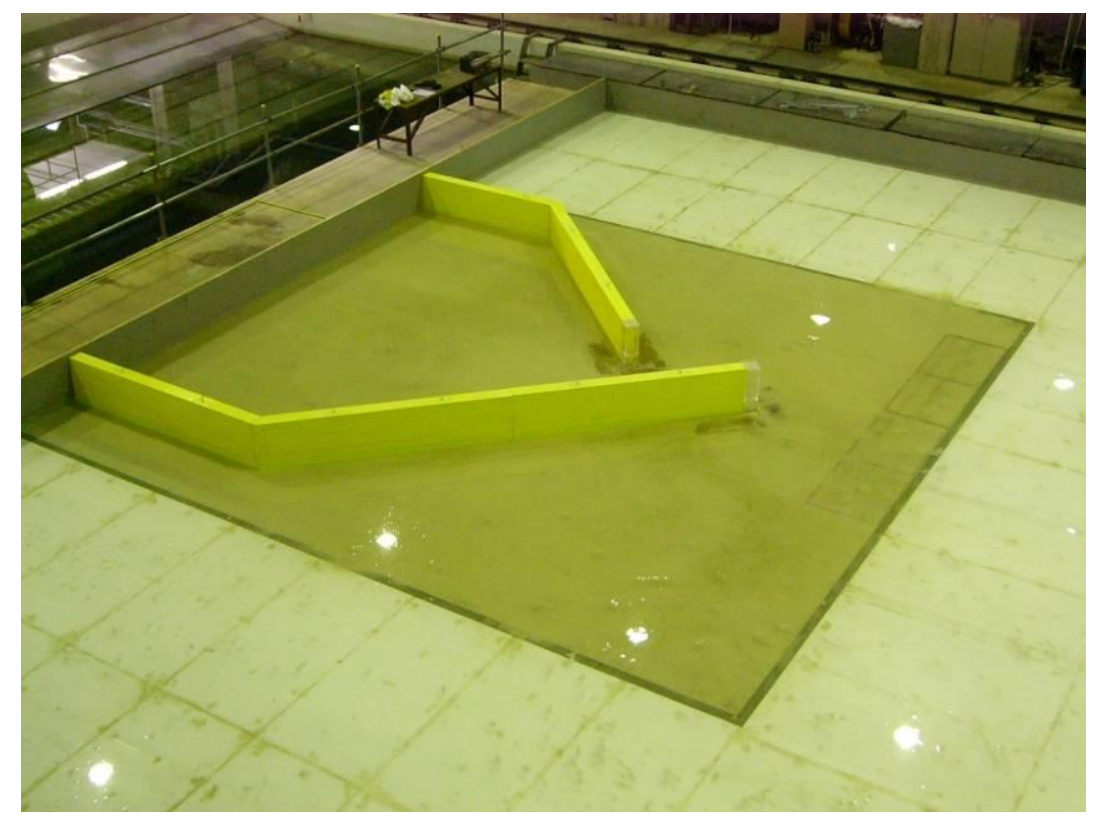

Figure 7. The harbor model used in the laboratory experiment. The size of the harbor was approximately $6.0 \mathrm{~m}$ by $6.0 \mathrm{~m}$. The typical harbor shape was applied in this study. 

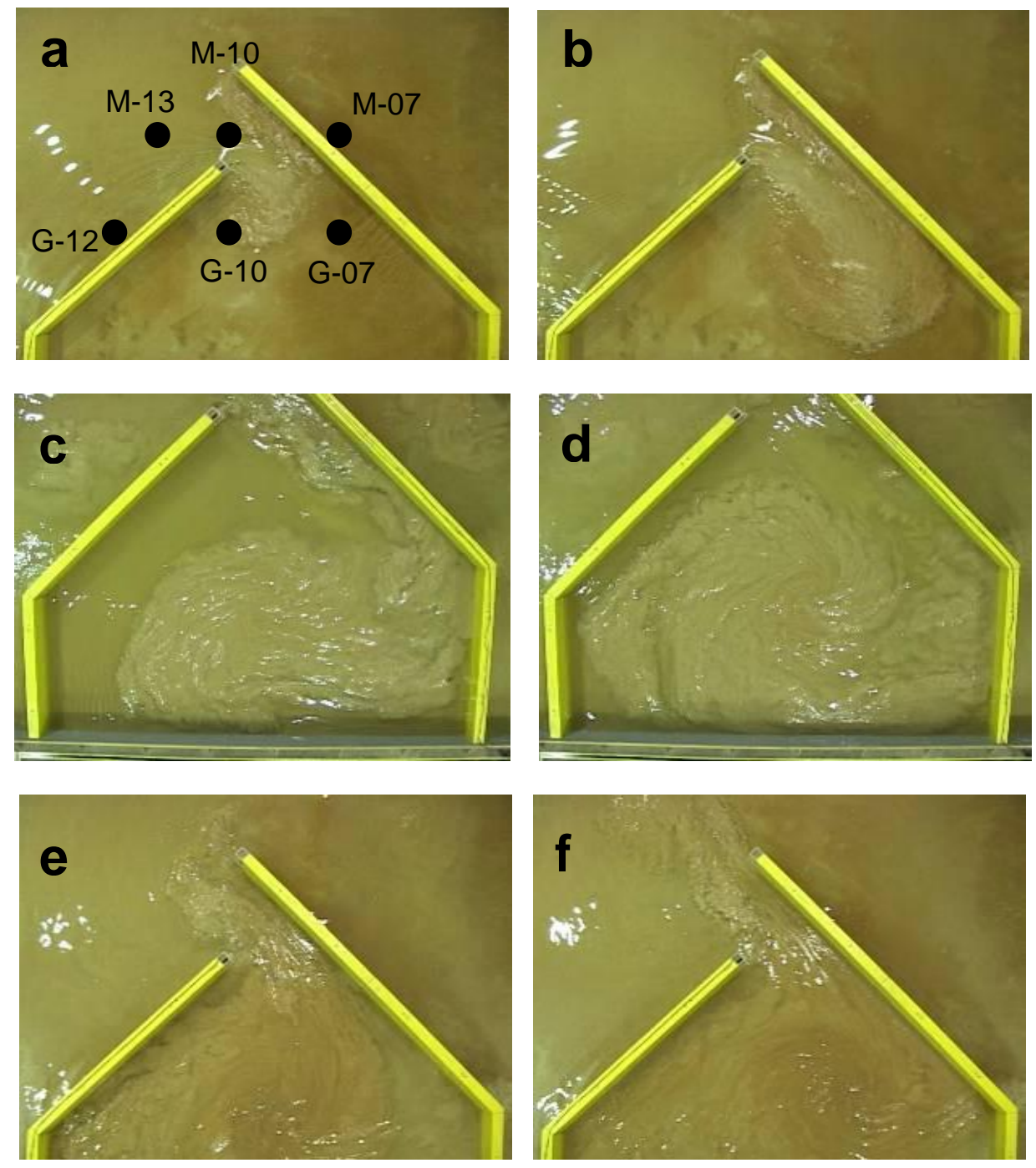

Figure 8. The vortex around the harbor. The vortex was appeared at the mouth of the harbor when the tsunami entered into the harbor $(a, b)$ and it migrated into the harbor, as a result one large vortex was made in the harbor (c, d). The vortex disappeared when the water level decreased and the entrainment of the bed sand was observed at the entrance(e, $f)$.

harbor locates through the 1/10 slope of $2.5 \mathrm{~m}$ length and 1/13 slope of $6.0 \mathrm{~m}$ length. The water depth at the plane part was $8 \mathrm{~cm}$. We assume that this model represents the harbor with scale of $1 / 100$. The topography change after the experiment was measured using laser displacement sensors. The spatial interval of the topography measurement in the longshore direction was $0.1 \mathrm{~m}$ (most area) and $0.05 \mathrm{~m}$ (around the entrance). The experiment was conducted twice at the same condition in order to check the reproducibility of the experiment.

To obtain the flow field and horizontal distribution of the water elevation change, we also conducted the experiment on the fixed bed. In this experiment, the movable bed was replaced by mortar floor. The wave gages and velocity meters were set outside and inside of the harbor. We measured the data at intervals of $0.5 \mathrm{~m}$ in the most of the area, of $0.25 \mathrm{~m}$ around the mouth of the harbor.

Figure 8 shows some pictures of characteristic flow field made in the experiment. A vortex appeared around the breakwater when the incident wave reached the entrance of the harbor (Figure 8 a, b). It was observed that the vortex entrained the bottom sand around the edge of the breakwater and caused highly turbid water. The vortex migrated into the harbor with turbid water and grew larger. As a result, the one large vortex centered at the central position of the harbor existed for approximately 10 seconds (Figure $8 \mathrm{c}, \mathrm{d}$ ). When the water level decreased, the return flow carried much suspended sand to the outside of the harbor. The return flow along the main breakwater and the vortexes appeared at the edge of the interior breakwater obviously entrained the bottom sand (Figure $8 \mathrm{e}, \mathrm{f}$ ). 

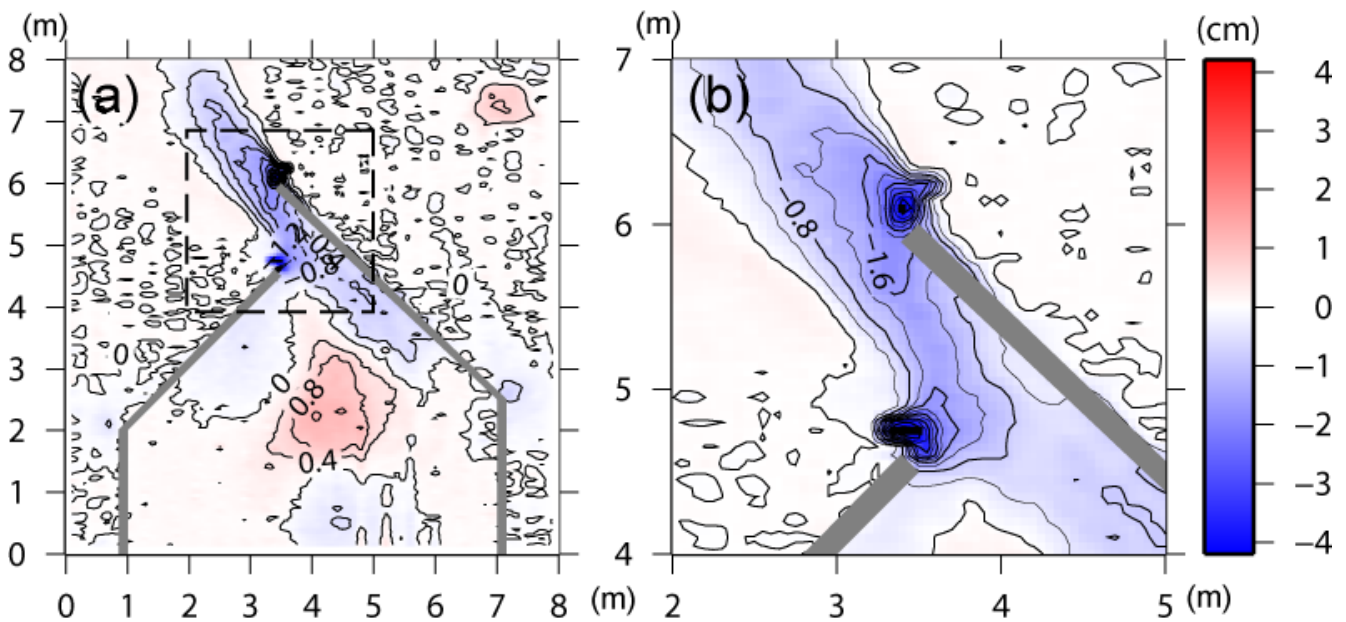

Figure 9. Topography change measured in the experiment. The remarkable erosion was made at the entrance of the harbor (b). In the harbor, the topography change is basically deposit (a). The maximum deposition appeared at the central part of the harbor, which corresponds to the center of the vortex (figure 8(d)).

The significant erosion was made at the entrance of the harbor (Figure 9). In the harbor, the deposition of the sediment appeared at the center of the harbor which correspond to the center of the vortex located. The topography change does not show a large difference between the two experimental cases.

\section{Comparison between the experimental result and numerical results}

The numerical calculation using the proposed pick-up rate formula was applied to the laboratory experiment on the topography change around a harbor. The numerical simulation was conducted using two pick-up rate formulae; Takahashi's formula (Takahashi et al., 2000) and the presented formula.

The conditions of the numerical calculation were presented in table 1 . The fluid motion was calculated using the non-linear shallow water equation (Takahashi et al., 2000). The two layered sediment transport model was adopted to calculate the topography change. The topography change was calculated using the following equation:

$$
\frac{\partial z_{b}}{\partial t}+\frac{1}{1-\lambda}\left(\frac{\partial q_{B x}}{\partial x}+\frac{\partial q_{B y}}{\partial y}-P+w_{s} C_{b}\right)=0,
$$

where $z_{b}$ is the height of the sand bed, $\lambda$ means the porosity, $q_{B x}, q_{B y}$ indicate the bed load in each direction, respectively. The suspended sediment is calculated by

$$
\frac{\partial \bar{C}(h+\eta)}{\partial t}+\frac{\partial \bar{C} M}{\partial x}+\frac{\partial \bar{C} N}{\partial y}-P+w_{s} C_{b}=\frac{\partial}{\partial x}\left(K_{h} \frac{\partial \bar{C} D}{\partial x}\right)+\frac{\partial}{\partial y}\left(K_{h} \frac{\partial \bar{C} D}{\partial y}\right)
$$

where, $\overline{\boldsymbol{C}}$ means the average sediment concentration in the suspend load layer, $M$ and $N$ are the fluid flow in each direction obtained by the non-linear shallow water equation, and $K_{h}$ indicates the horizontal diffusion coefficient, respectively.

The bed load formula and pick-up rate formula is different between two numerical simulations in order to reproduce the numerical condition which the sediment transport rate formula was derived and validated. The following bed load formula (Takahashi, 2000) was used for the calculation using Takahashi's formula.

$$
q_{B} / \sqrt{s g d^{3}}=21 \psi^{3 / 2} .
$$

Ashida-Michiue (1972)'s bed load formula was adopted for the numerical experiment with the presented pick-up rate formula. Yoshii et al (2009) have reported that the prediction using AshidaMichiue's formula is in good agreement with the measured bed load in the experiment. 


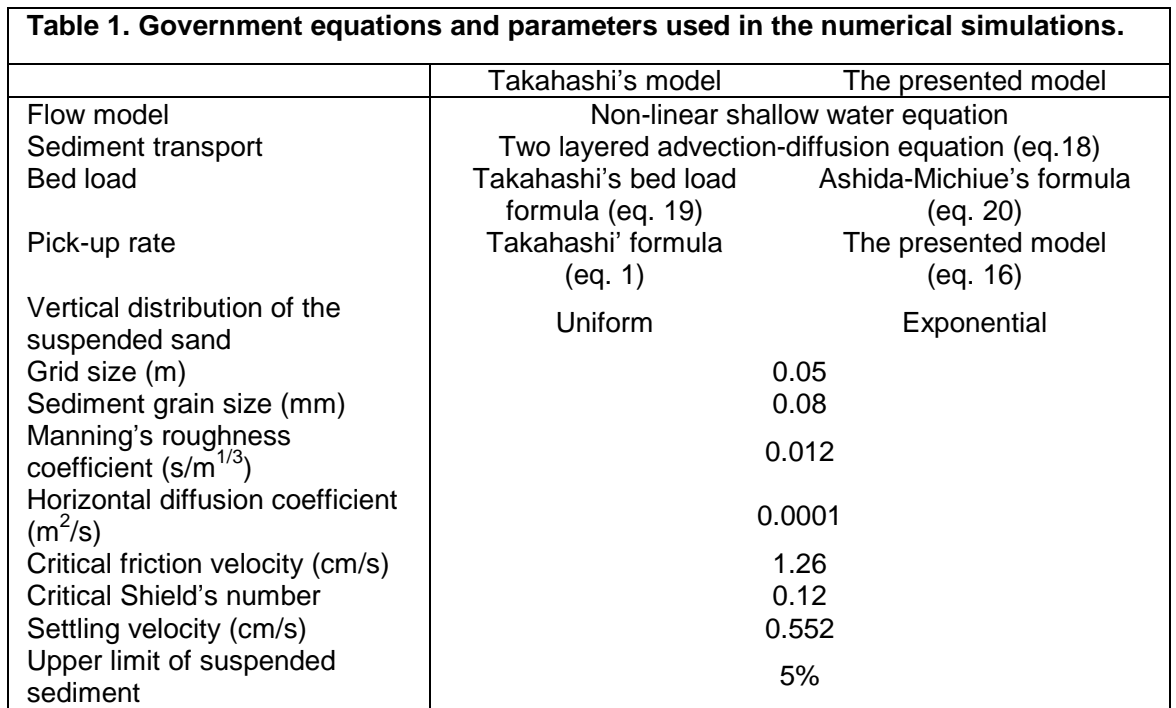

(n)

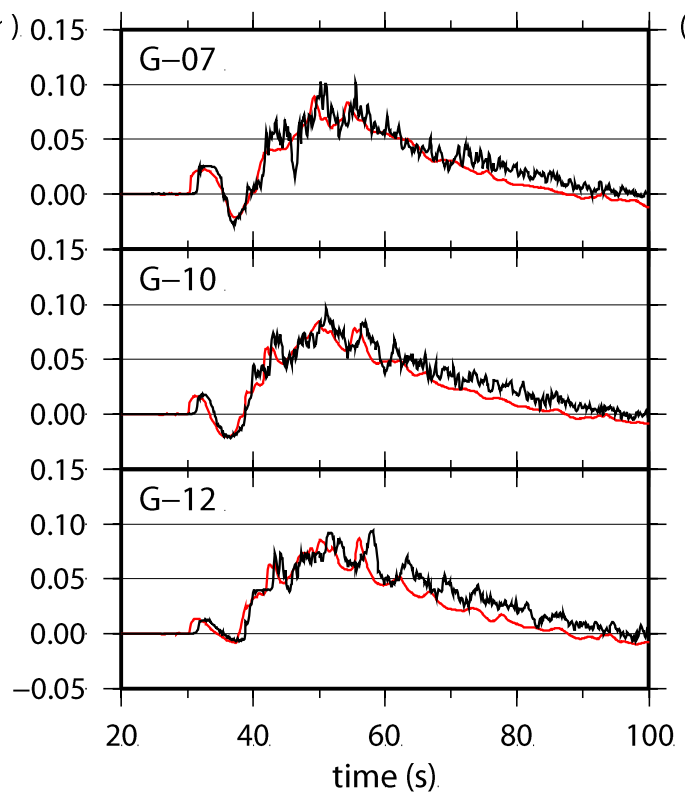

(n) 0.15

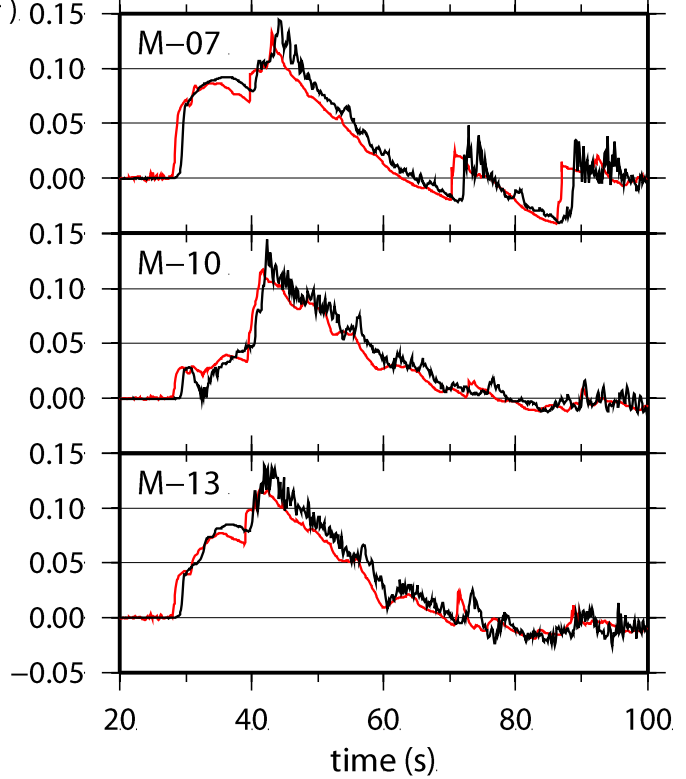

Figure 10. Time history of the water elevation measured in the laboratory experiment and calculated. Each figure shows water elevation presented in figure 8 . The solid lines in black show the measured water level and the solid lines in red indicate the calculated one.

$$
q_{B} / \sqrt{s g d^{3}}=17 \psi^{1.5}\left(1-\frac{\psi_{c}}{\psi}\right)\left(1-\frac{u_{b_{c}}^{*}}{u_{b}^{*}}\right)
$$

From the depth-averaged sediment transport, the average sediment concentration is obtained. To calculate the sediment concentration at the bottom from the average concentration, we have to assume some kind of vertical distribution in the sediment concentration. In Takahashi's model, the average sediment concentration in the suspend sediment layer was used as the sediment concentration at the bottom. In the presented model, the bottom concentration was calculated assuming that the vertical distribution of the suspended sediment concentration can be fitted with exponential function based on the experimental result of Yoshii et al. (2009). The vertical distribution of the suspended sediment concentration is expressed by 


$$
C(z)=C_{b} \exp \left(-\frac{w}{k_{z}} z\right),
$$

where, $k_{z}$ means the vertical diffusion coefficient . The vertical diffusion coefficient was determined by

$$
k_{z}=\kappa u_{*}(h+\eta) c_{k},
$$

where $\kappa$ is Karman's constant $(=0.4)$ and $c_{\mathrm{k}}$ is an undetermined coefficient. In this study, we used 0.2 for $c_{k}$ according to Fujii (1998). From equation 19, the concentration at the bottom is given by
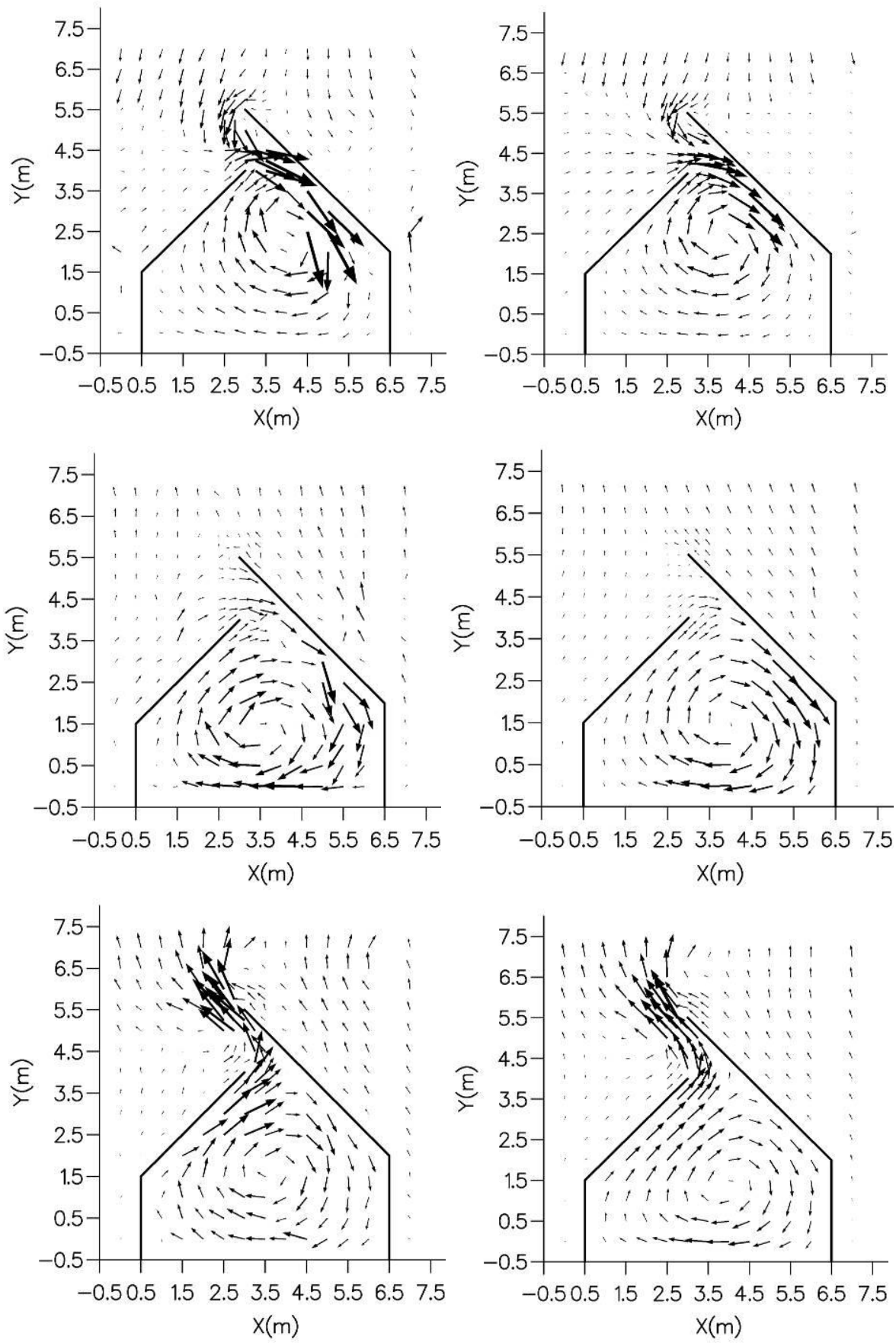

(a) numerical result

(b) experimental result

Figure 11. The spatial distribution of the horizontal current. 


$$
C_{b}=\frac{w(h+\eta)}{\left[1-\exp \left\{-w(h+\eta) / k_{z}\right\}\right.} \bar{C} .
$$

The settling velocity was calculated using Ruby (1933)'s formula for both simulations. The amount of sand settling down was obtained by multiplying the settling velocity by the sediment concentration at the bottom. Two layered sediment transport model sometimes leads the unrealistic amount of suspended sediment by supplying much sand from the bottom (Asai et al., 1999). To prevent such distant situation, the maximum concentration of suspended sand was adopted. If the sediment concentration reaches the maximum limit, the pick-up of the sand balanced with the amount of the settling sand.

Figure 10 shows the water elevation measured in the experiment and calculated water elevation around the harbor. The time history of the surface water elevation calculated and measured coincide with each other. The numerical result using the proposed formula consists with the experimental result and shows an advantage in all the compared regions over the conventional formula. Figure 11 shows the spatial distribution of the flow measured and calculated. The numerical simulation is successful in reproducing the flow around the harbor though the magnitude of the current is slightly different in some spots. From this result, the flow field around a harbor due to tsunami is believed to be reproduced by non-linear shallow water equations with sufficient level.

The sediment transport and topography change was calculated using the flow field calculated by non-linear shallow water equation. Figure 12 shows the measured topography change and the calculated topography change with each model. In this study, we calculate the topography change with varying the

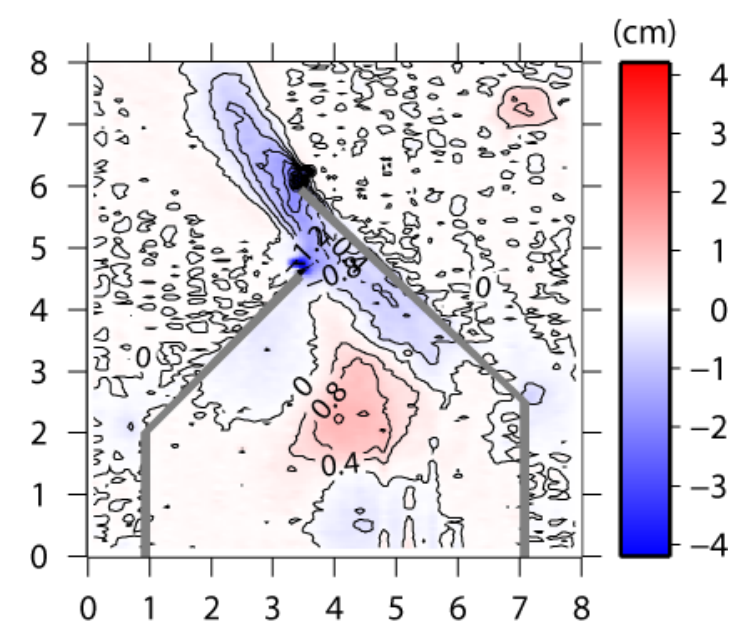

(a) Experiment

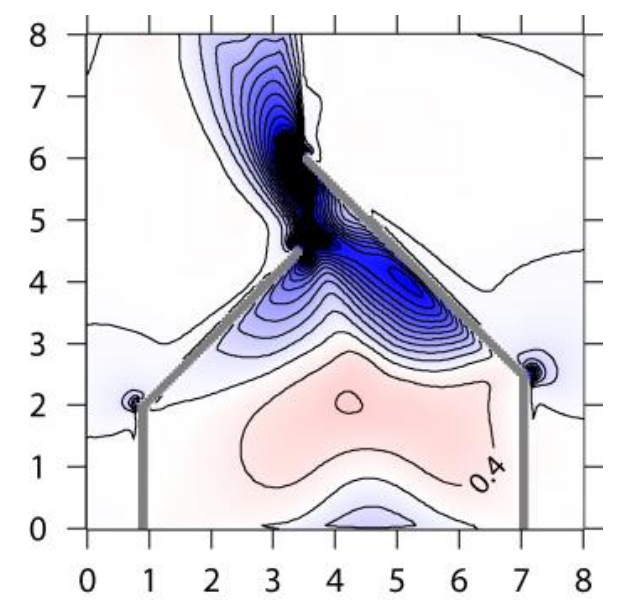

(b) Takahashi's model

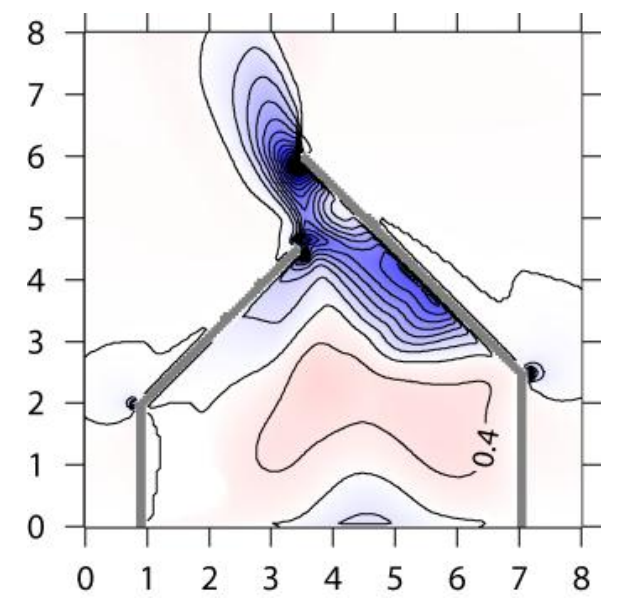

(c) The presented model

Figure 12. Topography due to tsunami around the harbor. The positive value means the deposition and the negative value means the erosion. The presented model was calculated with 0.15 of $F(s)$. 
coefficient of $F(\mathrm{~s})$ from 0.1 to 0.2 in equation 16. Figure 12 (c) shows the numerical result with 0.15 of $F(\mathrm{~s})$, which is the most similar to the experimental result.

Both models can reproduce the erosion at the entrance and the deposition at the center of the harbor. This result is considered to significantly depend on the accuracy of the reproduction of the water motion. However, the amount of topography change considerably differs in the two different sediment transport model. The result of Takahashi et al. (2000)'s model shows the remarkable erosion at the entrance of the harbor although the maximum limit of the suspended sediment concentration was adopted. Furthermore, the area of the sand deposition in the harbor was wider than the experiment. This might be due to the nimious supply of the sand from the significant erosion. As mentioned above, Takahashi et al. (2000)'s model is based on the experimental result of $0.2 \mathrm{~mm}$ sand. Thus, this model tends to overestimate the pick-up rate of $0.08 \mathrm{~mm}$ sand (Figure 5). Thereby, the numerical calculation using Takahashi et al. (2000)'s model results in the overestimation of sand transport.

On the other hand, the result of the presented model relatively agrees with the experimental result although the amount of the deposition at the center of the harbor is slightly underestimated. The maximum concentration of suspended sand is less than $2.0 \%$, which is smaller than the limit of concentration. If the average sediment concentration was treated as the concentration at the bottom as is the case with Takahashi et al. (2000)'s model, the amount of the sedimentation at the center was reduced. Therefore, the adoption of the vertical distribution of the suspended sediment concentration seems to contribute the accurate estimation of the amount of settling sediment. Kihara and Matsuyama (2009) and Sakakiyama et al. (2010) indicated the significance of the secondary current near the bottom which streams in the center of the vortex for the deposition at the center of the vortex. The underestimation of the deposition might be due to the un-consideration of such three-dimensional current component.

Figure 13 shows amount of topography change with the ratio of amount of erosion calculated to that measured in five regions presented in figure 13(a). Takahashi et al. (2000)'s model obviously overestimates the amount of deposition and erosion compared to the presented model. The presented model shows better agreement than Takahashi et al. (2000)'s model even if the coefficient of $F(\mathrm{~s})$

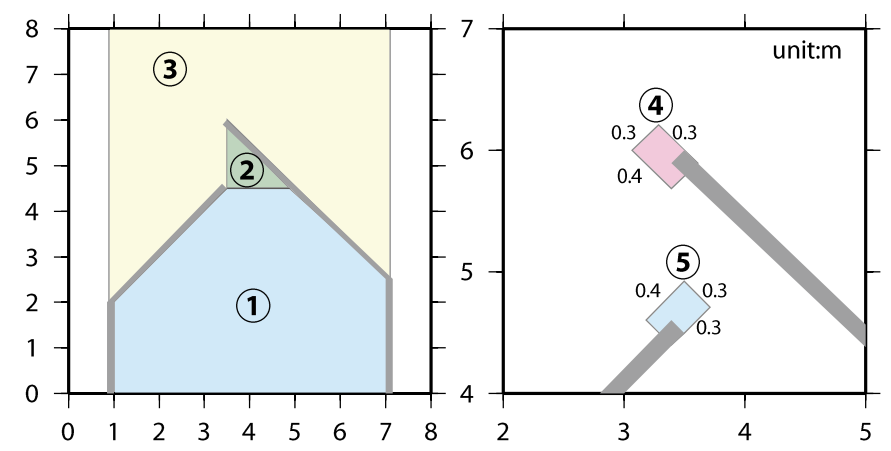

(a) The areas for the comparison of topography change

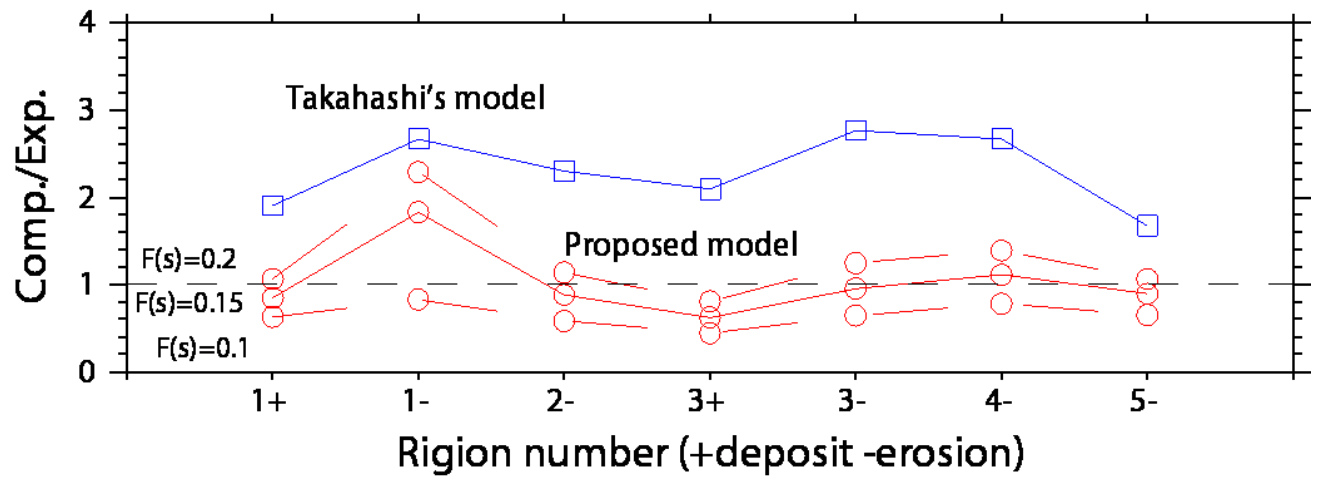

(b) The amount of deposit and erosion at the areas presented above.

Figure 13. Comparison between the experimental topography change and computational one. 
varies from 0.1 to 0.2 . The coefficients which shows the best agreement is 0.15 . Therefore, from the results discussed in this study, the coefficient of 0.15 is the most recommended. Finally, we propose the following equation as pick-up rate formula which can adopted to wide-range grain size and to topography change due to tsunami.

$$
P / \sqrt{s g d}\left(s g d^{3} / v^{2}\right)^{0.2}=0.15\left(\left(w_{s} / \sqrt{s g d}\right)^{0.8}\left(\psi-\psi_{c r}\right)\right\}^{2} .
$$

Adopting the settling velocity calculated by Rubey (1954) with $0.2 \mathrm{~mm}$ grain size $(=2.52 \mathrm{~cm} / \mathrm{s})$ to equation 24 , the proposed equation is transformed and the following equation is obtained as the pick-up rate formula for $0.2 \mathrm{~mm}$ sand.

$$
P / \sqrt{s g d}=0.015\left(\psi-\psi_{c r}\right)^{2} .
$$

The coefficient of 0.015 is similar value to that used in Takahashi's formula $(=0.012)$. Therefore, the proposed formula is compatible with the conventional formula for $0.2 \mathrm{~mm}$ sand. Though, if the grain size differed from $0.2 \mathrm{~mm}$, the proposed formula can predict the more accurate pick-up rate.

\section{Conclusion}

In this study, the authors investigated the pick-up rate through the dimensional analysis and laboratory experiments. The dimensional analysis revealed that three non-dimensional parameters should be considered in the pick-up rate formula. The must-considered non-dimensional parameters are "non-dimensional sand grain diameter", "non-dimensional settling velocity", and "non-dimensional shear stress". The pick-up rate formula including these parameters was presented. From the comparison between the pick-up rates measured in the experiment and that calculated using the proposed formula, it was confirmed that the proposed formula can explain the effect of grain size on the pick-up rate. The topography change, horizontal velocity and water elevation due to tsunami around the typical harbor was measured in the laboratory experiment. The numerical model which used the proposed formula showed the better reproducibility compared to that with conventional pick-up rate formula.

\section{ACKNOWLEDGMENTS}

The present study was conducted as a part of the activity of the Tsunami Evaluation Subcommittee (Chair person: Prof. Shuto) of the Nuclear Civil Engineering Committee in JSCE (Japan Society of Civil Engineers), which was supported by Japanese electric power companies. The authors would like to express their deepest gratitude to the members of the subcommittee. The authors also appreciate Prof. Takahashi and Dr. Kuriyama for their comments on this study. The laboratory experiment described in this study was supported by Dr. Inagaki and Mr. Mukohara with Kajima Corporation. We appreciate their corporation.

\section{REFERENCES}

Asai, D., T. Takahashi and F. Imamura. 1999. Tsunami Sand Transport at Iruma Izu in the 1854 Tokai Earthquake, Proceedings of the Annual Conference in H10, Tohoku Branch of JSCE, pp.266-267. (in japanese)

Ashida, K. and M. Michiue. 1972. Study on hydraulic resistance and bed-load transport rate in alluvial streams, Proc. Japanese Society of Civil Engineers, No.206, pp.59-69. (in japanese)

Iwagaki, Y. 1956. (I) Hydrodynamical study on critical tractive force, Transaction of the Japanese Society of Civil Engineers, Vol. 41, pp.1-21. (in japanese)

Kihara, N. and M. Matsuyama. 2010. Three-dimensional hydrostatic numerical simulations on tsunamiinduced sediment transport in a model harbor, Abstracts of the 3rd International Tsunami Field Symposium, p31-32.

Nishihata, T., Y. Tajima, T. Moriya and T. Sekimoto. 2006. Topography change due to the Dec 2004 Indian ocean tsunami - field and numerical study at Kirinda Port, Sri Lanka -, Proceedings of the 30th International Conference on Coastal Engineering, Vol. 2, pp. 1456-1468.

Rubey, W.W. 1933. Settling velocities of gravels, sand and silt particles, American Journal of Science, Vol. 25, pp.325-338. 
Sakakiyama, T., M. Matsuyama and T. Yoshii. Experimental Study on Bottom Topography Change in Harbor due to Tsunami, Proceedings of the 30th International Conference on Coastal Engineering. (in press)

Takahashi, T., N. Shuto, F. Imamura and D. Asai. 2000. Modelling sediment transport due to tsunamis with exchange rate between bed load layer and suspended load layer, Proceedings of International Conference on Coastal Engineering 2000, Vol.2, ASCE, pp.1508-1519.

Van Rijn, L.C. 1986. Mathematical modeling of suspended sediment in nonuniform flows, Journal of Hydraulic Engineering, Vol. 112, pp.433-455.

Yoshii, T, M. Ikeno and M. Matsuyama. 2009. Experimental study of sediment transport caused by tsunami, Proceedings of Coastal Dynamics 2009, Paper No. 32. 